\title{
THE GROWTH OF DISTRIBUTED GENERATION IN GREAT BRITAIN AND ASSOCIATED CHALLENGES Samuel J Gordon ${ }^{1 *}$, Connor McGarry ${ }^{l}$, Keith Bell ${ }^{l}$
}

\author{
${ }^{1}$ University of Strathclyde, Department of Electronic and Electrical Engineering, Glasgow, UK \\ *Samuel.gordon@strath.ac.uk
}

\author{
Keywords: DISTRIBUTED GENERATION, ACTIVE NETWORK MANAGEMENT, SYSTEM \\ OPERATION, SYSTEM RESILIENCE.
}

\begin{abstract}
Great Britain has reached high penetrations of intermittent Distributed Generation (DG), which has influenced many aspects of power system planning and operation. There has been a lack of technical requirements for DG to provide grid support and for network operators to monitor, control and gather detailed information of DG installations. The work reported in this paper has explored available data sources to provide better visibility of the amount, size and type of DG installed at various locations in the distribution networks and evaluated it alongside whole electricity system trends. Several of the issues associated with a high volume of generation being unobservable and uncontrollable are surveyed and discussed, such as active network management and coordination of transmission and distribution ( $\mathrm{T} \& \mathrm{D})$ networks. Finally, the paper discusses system resilience, emphasising that DG loss-of-mains protection continues to present a threat to system security and DG adds uncertainty to the effectiveness of automatic defence mechanisms. The latter issues were brought into focus following a system disturbance in GB on August 9th, 2019 and are reflected on in this study.
\end{abstract}

\section{Introduction}

The motivation to decarbonise energy systems and the cost reduction of renewable energy technologies has significantly increased the share of electricity production taking place at the distribution level, offering a progressively more decentralised energy system. The increased use of Distributed Generation (DG), such as wind and solar and other Distributed Energy Resources (DERs), such as energy storage devices, alters power flows within electricity distribution networks and may require changes in the electric power system operation and architecture.

High penetration of variable DG presents several challenges to power system planning, operation and control, including but not limited to, accommodating DG capacity and increased constraint management of distribution networks, increasingly localised supply resulting in a less schedulable generation portfolio for the Transmission System Operator (TSO) and increased inverter fed generation providing reduced system inertia and changing system dynamics [1].

Denmark is an early example of a power system with high amounts of DG, reaching over $50 \%$ of installed capacity by 2007. At this time, an innovation project in hybrid network control was launched by the TSO to address power system security problems associated with high DG [2]. Similar issues are being experienced in Great Britain (GB) today and discussed in this report. In Spain in 2006, the TSO commissioned a dedicated control centre which receives realtime communication of active power produced by generators $>1 \mathrm{MW}$. This has led to $99 \%$ of wind and $70 \%$ of PV capacity being observable, and plants of $>10 \mathrm{MW}$ being able to respond to active power set points [3]. Germany also has a significant penetration of intermittent DG, leading to constraints at various voltage levels, much of this DG is installed at lower voltages and not monitored. Curtailment of DG is possible by the German TSOs. However, it requires well-coordinated communications as they must contact the various regional and medium voltage control centres to make a request for manual adjustment [3].

Some areas of GB have now achieved high penetration of variable DG. Initially, technical rules were underdeveloped as connections to distribution networks in GB were made with a 'fit and forget' design approach and with an assumption of a strong transmission system. This led to shortcomings in DG technical requirements, including a lack of visibility and control, the under-exploitation of grid support functionality and ill-considered protection settings which can reduce power system resilience [4], [5]. In GB, there has also been a lack of common protocols for recording DG data leading to uncertainty in the amounts and types of DG at various locations in the network. Therefore, limitations arise when considering DG in operational processes, modelling and technical system studies.

Section 2 of this paper provides an assessment of the amounts of DG embedded within the GB power system. Section 3 reviews some of the many challenges for operating a power system with high DG. Section 4 discusses the impacts of DG on power system resilience, reflecting on existing issues exposed by a recent power system disturbance event in GB. Section 5 concludes the paper.

\section{Distributed Generation in GB: Type, size and location}

A key factor to study and plan for the efficient operation of a power system with high penetrations of DG is transparency of network data. There are three main sources of DG data in GB: (1) Future Energy Scenarios (FES) published by National Grid Electricity System Operator (NGESO) which outlines credible 
growth scenarios for the whole electricity system; (2) the Department for Business, Energy \& Industrial Strategy (BEIS) who publish historic energy data and trends; and (3) Long Term Development Statements (LTDS) published by Distribution Network Operators (DNOs) for each licence area. Each data source is created by a different organisation with different intentions. When compared, only partial correlation can be achieved and variation is observed in installed capacities, technology groupings and the granularity of data, making it problematic to combine features from each data source.

The LTDS are part of a DNO's distribution licence conditions with the primary function of providing data for developers to assess and evaluate local connection opportunities. It has been recognised by Ofgem, the regulator for GB, that improvement to the LTDS is core to enhancing the availability of network forecasting and planning data and will underpin several functions to enable the DNO to transition to a Distribution System Operator (DSO) [5]. Currently, the LTDS is reported in a common overall format but is generated independently by each of the 14 DNO licence areas in GB. However, the contents of each LTDS shows many inconsistencies between licence areas, in particular with labelling and descriptive detail.

\subsection{Current DG Installations}

Fig. 1 shows DG, connected to all DNO licence areas in GB and split by technology type, as a proportion of total registered installed generation capacity, according to the LTDS data and the Transmission Entry Capacity register as of 2019 [6]. As the LTDS does not include installations less than $1 \mathrm{MW}$, data for micro PV installations from BEIS have been included [7], with the assumption that PV makes up the vast majority of small installations. 'Embedded' generation is connected to the distribution network yet has contracted with National Grid to obtain rights of access to the transmission network and is therefore 'visible and controllable'. DG, which makes up 35\% of total installed generation capacity, is largely unobservable and uncontrollable by the TSO. $59 \%$ of DG is asynchronous with an intermittent renewable source and, while the remaining $41 \%$ is synchronous generation with a solid fuel source, it lacks the plant level and network level controls to be utilised for system operation.

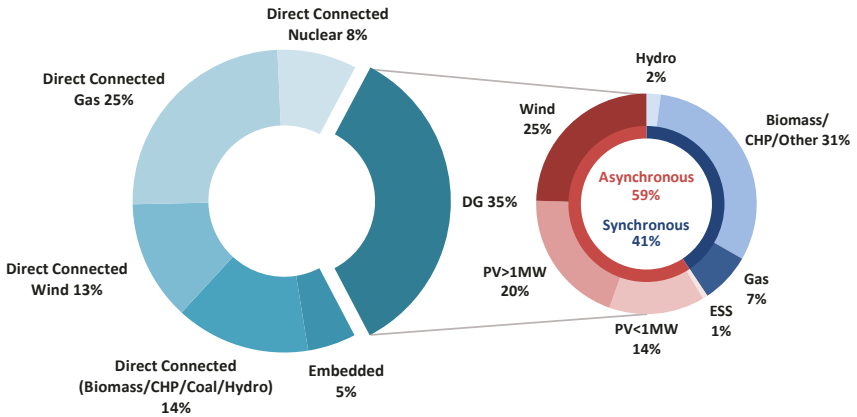

Fig. 1. Generation mix in GB transmission and distribution networks.

Table 1 shows the increase in the number of projects at lower distribution voltages. Registered DG connections above $1 \mathrm{MW}$ connected at $11 \mathrm{kV}$ or below make up $23 \%$ and $8 \%$ of distribution connected and total connected capacity,

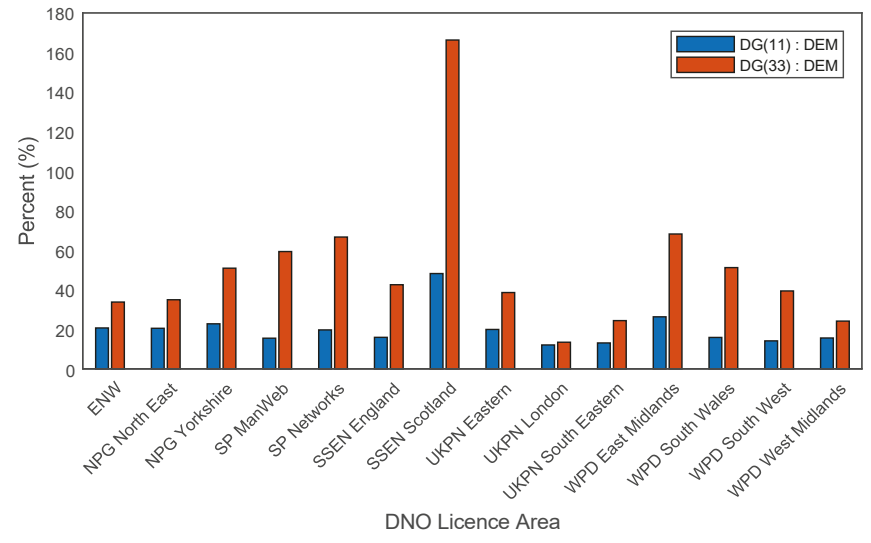

Fig. 2. Ratio of installed DG capacity at $11 \mathrm{kV}$ and $33 \mathrm{kV}$ to sum of DNO peak substation demands.

respectively. However, they represent $57 \%$ of the total number of sites. As the connection voltage level increases, the mean project size increases and the number of connections reduces significantly. This information exposes the extent of the challenge of improving visibility within distribution networks, with a sizeable amount of capacity located across numerous sites at lower distribution voltages.

For the sub-1 MW category, the mean capacity of each site is small due to the volume of installations on residential and commercial buildings. According to BEIS data for solar PV deployment [7], by December 2019 a total of 1,000,565 solar PV installations exist, which make up 13,358 MW of PV capacity. Of this, $99.5 \%$ of installations are less than $50 \mathrm{~kW}$, equating to 3,730 MW of capacity. However, this data cannot be correlated with the LTDS data through lack of consistency when categorising project capacity.

Table 1: Project size at different voltage levels (LTDS)

\begin{tabular}{lcclc}
\hline & $\begin{array}{l}\mathrm{DG} \leq \\
11 \mathrm{kV}\end{array}$ & $\begin{array}{l}11 \mathrm{kV}< \\
\mathrm{DG} \leq \\
33 \mathrm{kV}\end{array}$ & $\begin{array}{l}33 \mathrm{kV}< \\
\mathrm{DG} \leq \\
132 \mathrm{kV}\end{array}$ & $\begin{array}{l}\text { Trans- } \\
\text { mission }\end{array}$ \\
\hline \hline $\begin{array}{l}\text { No. of sites } \\
(>1 \mathrm{MW})\end{array}$ & 1,902 & 1,175 & 127 & 152 \\
$\begin{array}{l}\text { Total installed } \\
\text { capacity (MW) }\end{array}$ & 7,385 & 16,600 & 8,297 & 65,683 \\
$\begin{array}{l}\text { Mean Capacity } \\
(\mathrm{MW})\end{array}$ & 3.9 & 14.1 & 65.3 & 432.1 \\
\hline
\end{tabular}

The higher the ratio of DG to local demand, the greater impact DG has on the wider system due to (1) a reduction in net demand seen by the transmission system, (2) an increasing occurrence of 'reverse power flow' supplies to the transmission network and (3) increased variability in net transmission demand as true demand is being supplied by variable DG. Fig. 2 shows the ratio of installed DG to demand where DG(11) is all generation connected at $11 \mathrm{kV}$ and below and DG(33) is all generation connected at $33 \mathrm{kV}$ and below. The ratio considers total installed capacity for DG and combined annual peak of all primary substation demands. Diversity in both demand and generation is not considered and is therefore intended only as a guiding metric to identify DNO licence areas with high DG. Fig. 3 shows the technology split at different voltage levels. 
Visibility of power flows within distribution networks, particularly in $11 \mathrm{kV}$ and LV networks has historically been poor, partly due to lack of need. However, growth in peak demand, network complexity and decentralisation calls for greater visibility across network voltage levels, which is a challenge for current data systems and architectures [5], [8]. Furthermore, there are currently no common data sharing protocols or mechanisms for network monitoring data [5]. It is important that energy system data can be shared across platforms and meets the needs of multiple types of users, including the DSO to make decisions and open up flexibility markets, flexible service providers and consumers while respecting privacy and consumer protection.

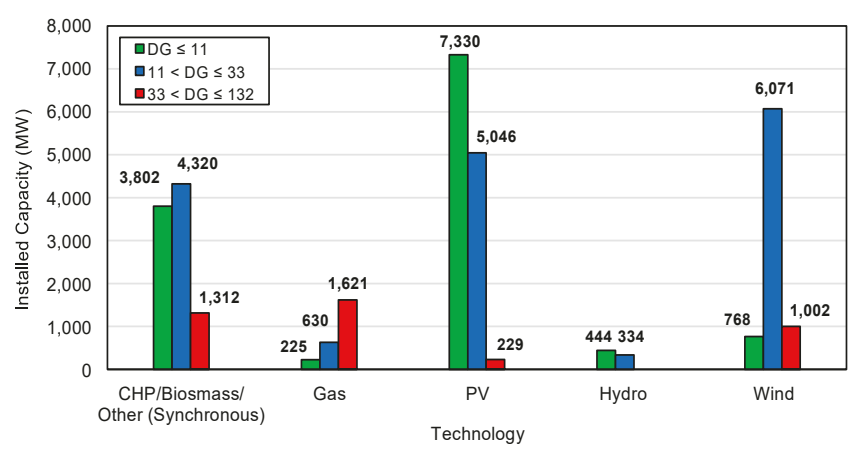

Fig. 3. Distributed generation technologies, installed at different distribution voltages in GB.

\subsection{Growth Trends for Distributed Generation}

Fig. 4 shows the installed capacity connected to the Transmission and Distribution ( $\mathrm{T}$ and $\mathrm{D}$ ) networks for the period 2011 to 2018 inclusive [9]. In the period between 2011 and 2019, DG grew substantially, which was stimulated by government incentives such as Feed-in Tariffs (FiT). Installed capacity on the distribution network grew by an average of more than $10 \%$ per year, driven by additions in asynchronous technologies, primarily intermittent wind and solar. In the same period, asynchronous generation also grew substantially on the transmission network, with significant additions of onshore and offshore wind connections, while both overall installed capacity and synchronous generation in the transmission network reduced following closure of thermal plant. This combination of factors results in a less schedulable and less dispatchable generation portfolio for the TSO.

To estimate future growth, the FES 2019 [10] Community Renewables (CR) and Two Degrees (TD) scenarios are considered, which represent an expected high uptake of distributed technologies and a more centralised generation portfolio respectively. These Future Energy Scenarios indicate that transmission connected generation will shift to growth and investment in new capacity from 2020 to 2050. Furthermore, both scenarios expect continued high growth for capacity connected to the distribution network.

The LTDS includes data for 'accepted' generation on each licenced network area. Accepted DG are those that have signed grid connection agreements with the $\mathrm{DNO}$, but projects may be on hold, e.g. due to lack of business case following withdrawal of subsidies. Several accepted projects may never get built. However, many could go ahead in the near term given the right market conditions. The accepted connections would increase DG capacity by $60 \%$ from $37,611 \mathrm{MW}$ to $60,836 \mathrm{MW}$. Of this, $29 \%$ would be at $\leq 11 \mathrm{kV}$ and $75 \%$ for 11 $\mathrm{kV}<\mathrm{DG} \leq 33 \mathrm{kV}$. The FES $2019 \mathrm{CR}$ and TD scenarios show installed capacity of DG to reach this figure by 2034 or 2043 respectively. Each year BEIS publish energy projections, including installed capacity for renewable energy. The forward projections for 2019 were $25 \mathrm{GW}$ in $2011,41 \mathrm{GW}$ in 2015 and $44 \mathrm{GW}$ in 2018 [11]. However, capacity for renewable energy at end of 2019 stands at $47.4 \mathrm{GW}$ [12], showing that, given the right economic and political enabling environment, rapid growth in the renewable energy sector can continuously surpass long term growth forecasts.

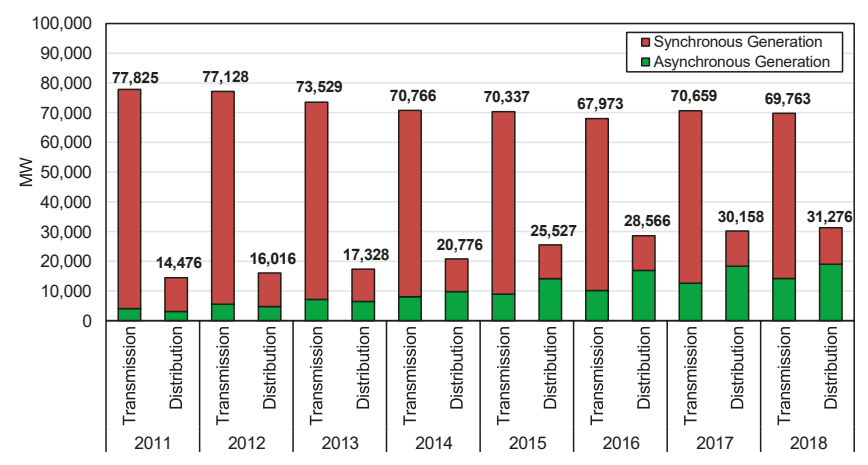

Fig. 4. Installed generation capacity, split into synchronous and asynchronous generation connected to the transmission and distribution networks in GB.

\section{Operational Challenges of an Increasingly Distributed System}

\subsection{Transmission System Operation}

National electricity system operation in GB is performed by NGESO. Due to the changing generation mix highlighted in Section 2, it is becoming increasingly challenging to balance the system during periods of low demand as it gets close to, and in some periods may fall below, the level of inflexible generation on the system [13]. DG can contribute towards transfer constraints across transmission boundaries, and the SO may be required to: (1) curtail the output of transmission connected, possibly low carbon generation, through the balancing mechanism (BM) and other balancing services; or (2) agree contracts with large generators to ensure they are synchronised and providing reactive power capability or inertia, even during times when they would have otherwise been uneconomical to run [13], [14]. The costs of these operational actions are ultimately passed through to customers.

Many balancing services are naturally provided by assets embedded within the distribution network, for example, increasing or decreasing demand. Conversely, the TSO's ability to increase or decrease generation has traditionally been accessed via larger, dispatchable transmission connected generators. However, a broader range of flexible DER are starting to participate in TSO balancing and ancillary service markets. These include the BM [15] as well as frequency response and reserve ancillary service markets previously restricted to participants in the wholesale electricity market, which now allow smaller entry capacities and aggregation [16], 
[17]. However, there are barriers to entry for DG which are mainly the result of a high cost of compliance with a number of different codes [18].

\subsection{Active Network Management}

To accommodate the growth of intermittent DG, Active Network Management (ANM) schemes are being deployed by DNOs in GB [19], [20]. To date, ANM connections are typically offered to generators applying to connect to constrained areas of the distribution network, where the DG connection would have otherwise triggered network reinforcements due to, e.g. thermal overloads. The relatively high cost of network reinforcement required to accommodate the DG project would largely be borne by the applicant and would often be prohibitively high. ANM connections allow generators to connect more quickly and at a lower cost by including provisions for curtailing their output during constrained periods. DG developers must accept the cost of unsold energy generation, coupled with the long-term risk that the business case could diminish over time due to changing system conditions impacting the level of curtailment. The authors in [1] argue that the DNO is best placed to manage the curtailment risk for ANM connectees, given that they are the only organisation with enough knowledge of the network to appropriately forecast and have control over network constraints. This could be achieved by the DNO paying towards the cost of lost energy through a price for curtailment contracted in the connection agreement.

\subsection{Visibility, Control and Coordination}

For the most part, procurement of flexibility exists to resolve the issues faced by each system or network operator, and much work remains in the move towards a 'whole electricity system' set of grid management services. Both the DNO and TSO have the potential to benefit from flexible resources located in distribution networks. However, enhanced observability of the significant amounts of DG in the system and coordination between the actions of both system operators is required. The potential for conflicting actions and the need for cooperation are widely recognised and highlight the necessity to improve observability of the distribution networks and better transparency of grid data [21].

ANM systems can exacerbate or create further challenges. The purpose of ANM connections are to increase DG penetration in already constrained distribution areas, which may already contribute towards transmission export constraints. However, the schemes operate without being visible to the TSO. While T\&D constraint conditions might coincide, for example high wind and low demand conditions, and an ANM action may benefit both constraints (e.g. curtailing distributed wind), technical correlation is uncertain without system coordination. Furthermore, the forecasting uncertainty of transmission constraints will be increased, as the TSO is unaware whether the net load observed includes or excludes DG located behind an ANM system [22]. An ANM action also has the potential to counteract a balancing instruction by the TSO. For example, the TSO requests an adjustment from an embedded service provider, an ANM system will observe the change in power flow across a distribution constraint being monitored and automatically alter the allowable output of the ANM connection thus offsetting the effect seen at the transmission system and potentially requiring the TSO to procure the response again, from elsewhere.

In [23], a Distributed Energy Resource Management System (DERMS) and ANM systems are proposed for UKPN's network to optimise the delivery of TSO services while managing conflict with ANM connections. This represents a DSO-led solution, where the TSO would provide signals to the DSO, such as information on constraints and the flexibility service volumes. In any case, the distribution constraint must still be respected. So, in some network conditions and where embedded service providers and ANM generators are located behind a constraint, the coordination will require some prioritisation and ranking to optimise the available capacity. While this ranking should yield an overall system benefit, it will inevitably affect the business model of either the existing service provider by not being dispatched due to insufficient headroom, or the ANM generator, through increased curtailment to make space for the service provider.

As traditional means of voltage control from synchronous generators decreases, and load patterns change due to the high penetration of intermittent DG, new approaches for voltage control are required [3]. To address the need for voltage control at the T/D interface, a similar challenge exists to determine the amount of available reactive power at the interface node [24]. This requires additional visibility and control at the DG plant level but must also be simultaneously coordinated with transformer tap changer control to achieve the desired effect at the transmission system. Under G59 regulations, DGs in Britain were not required to install power plant controllers, capable of regulating each inverter and provide voltage droop at the point of common coupling. Projects are ongoing to trial participation from DGs to control voltages and address transmission system constraints [25]. DG will require reimbursement of costs for any lost active power due to limits of converter ratings and financial incentives to make changes to plant controls. In the past these services may have been provided as a mandatory Grid Code requirement for a large generator. A study to address voltage issues in the southwest of England indicates that dynamic voltage support from DGs would benefit the management of transmission faults. However, the only way to change the DGs power factor is to send an engineer to site to make a manual adjustment and is therefore highly unpractical and inflexible [26].

\section{Power System Resilience with High DG}

In recent years there has been much debate as to the definition of power system resilience. However, in general, the term power system resilience is encompassed by its ability to [27]:

- anticipate, prepare or tolerate disturbances,

- absorb, withstand or adapt to the disturbance,

- maintain functionality and recover quickly, and

- learn from the past.

Resilience is closely related to the terms reliability and stability, which are concerned with the ability to maintain an available system for services to be delivered and maintain 
stable operation following a disturbance, respectively. DG can impact power system stability and therefore can reduce its over-all resilience.

Currently $59 \%$ of DG in Britain is converter-fed, intermittent and asynchronous, which is expected to increase as highlighted in Section 2. There are many challenges associated with the operation of power systems with a high penetration of asynchronous power sources as they displace traditional synchronous machines and their inherent characteristics. These include, management of fault current and ensuring correct operation of protection systems and changing frequency dynamics resulting in higher rate of change of frequency (RoCoF) during a power imbalance [28]. The latter indicates that conventional frequency response services become unsuitable [29].

\subsection{Unintended Operation of DG Protection}

The planning of DG technical requirements and lack of visibility and control mean that DG has not traditionally contributed towards maintaining the stability of the system in the same way that larger generating units do. This is mainly attributed to DGs not being required to remain connected to the system during disturbances on the transmission grid. Loss of Mains (LoM) protection is designed to disconnect the DG and prevent unintentional islanded operation on the distribution network. The two original schemes and settings used in GB are based on the static sensing of RoCoF being $>0.125 \mathrm{~Hz} / \mathrm{s}$, or a voltage angle deviation of 6 degrees under Vector Shift (VS). In a low inertia system, protection devices with these settings may undesirably operate and trip DG off the system, even when the original fault is cleared, and cause cascade tripping of many small generating units resulting in high power imbalance [4]. Updates to G59/3 in 2015 stipulated that VS was banned and old RoCoF settings were not permitted on new or existing plant. However, DGs did not comply with updating legacy protection settings and it proved difficult to enforce onto generators. RoCoF is a determining factor for frequency behaviour and the $0.125 \mathrm{~Hz} / \mathrm{s}$ RoCoF limit becomes a dominant constraint when operating the GB system with low inertia. Therefore, the TSO must constrain down the largest infeed to maintain system security. This operational action will become increasingly uneconomical until the 'Accelerated LoM change programme' is completed to retroactively update DG protection settings, which is due in August 2022 [30].

\subsection{Low Frequency Demand Disconnection}

During extreme contingencies, i.e. those that are more severe than normal design contingencies, the scheduled frequency response holding may become insufficient to cover demand, causing frequency to fall outside of operational limits. To prevent reaching underfrequency protection limits of generating equipment and collapse of system frequency, the Low Frequency Demand Disconnection (LFDD) scheme automatically disconnects blocks of demand with the aim of restoring system frequency and allowing a new equilibrium to be reached. As DG penetration increases and demand is increasingly met locally, the effectiveness of the LFDD scheme is challenged, and its level of success becomes dependent on the demand and generation mix downstream of each relay, at the time of operation. Without visibility of DG, uncertainty is introduced into the amount of true demand on the system at any given time, and therefore, uncertainty in the net effect that automatic load shedding may have on the system frequency when activated. A study by NGESO in 2017 [31] investigated the effectiveness of LFDD with low system inertia and an increasingly high penetration of DG. Three scenarios with varying levels of DG were examined, the "current system" (as of 2017) with 13 GW of installed DG, and two future scenarios with $20 \mathrm{GW}$ and $30 \mathrm{GW}$ installed. The source of the DG estimates is not stated. However, the analysis of current LTDS data in this paper highlights that the most accelerated 'future system' assumption of $30 \mathrm{GW}$ installed DG has already been surpassed in 2019 , and that 13 GW was likely to be an underestimate of the installed capacity at the time. Therefore, the settings of the LFDD scheme requires further review in order to retain its effectiveness such that it can be relied upon in a wide range of system operating conditions.

\subsection{Reflections on August 9th, 2019 Disturbance Event}

A single lightning strike to an overhead transmission line on August 9th, 2019 lead to the combined loss of two large generators, Hornsea offshore wind farm and Little Barford gas plant, as well as a significant loss of DG due to the operation of VS, RoCoF or internal protection against under frequency. The total loss of power infeed from DG is estimated between $1300 \mathrm{MW}$ to $1500 \mathrm{MW}$ across the event. This can be compared to a total transmission connected generation loss of $1378 \mathrm{MW}$. Over the course of the event, frequency response and reserve holdings became exhausted and the system frequency dropped to $48.8 \mathrm{~Hz}$, triggering the first stage of LFDD and cutting supplies to 1.15 million customers [32]. The high penetration of DG had substantial ramifications during the August 9th event, by both contributing towards the loss of infeed, and reducing the net effect of the LFDD scheme. It is reported that 892 MW of demand was disconnected, yet the net demand reduction seen by the transmission system was reported to be $350 \mathrm{MW}$ [32]. This indicates that approximately $550 \mathrm{MW}$ of DG was disconnected by LFDD relay operation, which may normally be expected to require the TSO to procure and dispatch more power reserves to restore system frequency.

The event reveals key questions regarding how the system is operated and the uncertainties with high penetrations of DG. How much DG really tripped, and why? The amounts of DG lost, for which reasons and at which locations during the event are based on estimates by the DNOs. This is largely because, many years after significant volumes of DG started to be connected, the DNOs lack detailed monitoring of it. Is the "accelerated" programme to change LoM protection on DG by 2022 accelerated enough? And will it sufficiently remove the concern of DG tripping off the system unexpectedly? How much frequency response should be held, and how should intermittent $\mathrm{DG}$ be treated when considering response holdings?

\section{Conclusions}

This paper provides insight into the amounts and types of DG that is installed in GB. The lack of availability and transparency of consistent data for DG installations has been 
highlighted and argued to act as an obstacle to the development of more active distribution network operation and the use of flexibility from distributed energy resources. The issues described show the importance of understanding the role of distributed generation in the energy mix within the operation of an electricity system. Failure to adequately manage the operation of DG represents both a threat to the system and the missing of an opportunity to use the services that DG might provide. The risks of high volumes of DG which are neither observable nor controllable adds a significant amount of uncertainty to operational procedures and automatic defence mechanisms which can reduce system resilience.

\section{References}

[1] K. Bell and S. Gill, "Delivering a highly distributed electricity system: Technical, regulatory and policy challenges," Energy policy, vol. 113, pp. 765--777, 2018.

[2] P. Lund, "The Danish Cell Project - Part 1: Background and General Approach," in 2007 IEEE Power Engineering Society General Meeting, 2007.

[3] CIGRE Working Group C2.16, "Challenges in the control centre (EMS) due to distributed generation and renewables," 2017.

[4] A. Dyśko, D. Tzelepis and C. Booth, "Practical risk assessment of the relaxation of LOM protection settings in NIE Networks' distribution system," IET Generation, Transmission and Distribution, no. 15, p. 1335 - 1339, 2018.

[5] Ofgem, "Consultation: Key enablers for DSO programme of work and the Long Term Development Statement," 2019.

[6] NGESO, "Registers, reports, and guidance," [Online]. Available:

https://www.nationalgrideso.com/connections/registersreports-and-guidance. [Accessed 15 April 2020].

[7] BEIS, "National Statistics Solar photovoltaics deployment," [Online]. https://www.gov.uk/government/statistics/solar-

Available: photovoltaics-deployment. [Accessed 15 April 2020].

[8] G. Taylor, C. Axon and M. Irving, "Scalable high performance information and communications technology for smart distribution network operation," IEEE Power and Energy Society general meeting, pp. 1-8, 2012.

[9] BEIS, "Digest of UK Energy Statistics (DUKES): electricity, Table 5.12," 2019. [Online]. Available: https://www.gov.uk/government/statistics/electricitychapter-5-digest-of-united-kingdom-energy-statistics-dukes. [Accessed 2 May 2020].

[10] NGESO, "Future Energy Scenarios," [Online]. Available: http://fes.nationalgrid.com/fes-document/. [Accessed 2 May 2020].

[11] BEIS, "Energy and emissions projections," [Online]. Available:

https://www.gov.uk/government/collections/energy-andemissions-projections. [Accessed 2 May 2020].

[12] BEIS, "DUKES Section 6 - UK Renewables," 2020. [Online]. Available: https://www.gov.uk/government/statistics/energy-trendssection-6-renewables. [Accessed 5 May 2020].

[13] NGESO, "Summer Outlook," 2020.

[14] NGESO, "Regional Trends and Insights," 2018.
[15] NGESO, "Wider Access to the Balancing Mechanism Roadmap," 2018.

[16] NGESO, "Firm frequency response (FFR)," [Online]. Available:

Available: https://www.nationalgrideso.com/balancingservices/frequency-response-services/firm-frequencyresponse-ffr. . [Accessed 2 May 2020].

[17] NGESO, "Reserve services," [Online]. Available: https://www.nationalgrideso.com/industryinformation/balancing-services/reserve-services. [Accessed 2 May 2020].

[18] NGESO, "System Needs and Product Strategy," 2017.

[19] WPD, “Active Network Management," [Online]. Available: https://www.westernpower.co.uk/anm-further-info. [Accessed 2 May 2020].

[20] UKPN, "Flexible Connections," [Online]. Available: https://www.ukpowernetworks.co.uk/electricity/distributionenergy-resources/flexible-connections. [Accessed 2 May 2020].

[21] G. Helena, E. I. R. Puente and D. Six, "Coordination between transmission and distribution system operators in the electricity sector: A conceptual framework," Utilities Policy, vol. 50, pp. 40--48, 2018.

[22] Energy Networks Association, “Active Network Management Good Practice Guide," 2015.

[23] A. R. Ahmadi, M. Gordon, M. White and et al, "Enhanced Transmission and Distribution System Coordination and Control Utilising Distribution Network Capacity and Avoiding Conflicts of Service Offered to Transmission System Operator," in CIRED 2019, Madrid, 2019.

[24] J. Silva , J. Sumaili, R. Bessa and et al, "Estimating the active and reactive power flexibility area at the TSO-DSO interface," IEEE Transactions on Power Systems, vol. 33, no. 5, pp. 4741--4750, 2018.

[25] UK Power Networks, "Enhancing transmission and distribution system coordination and control in Great Britain using power services from distributed energy resources," in IET Renewable Power Generation, Copenhagen, 2018.

[26] Western Power Distribution, National Grid, “Joint network study for the south west transmission and distribution system," 2018.

[27] F. H. Jufri, W. Victor and J. Jaesung, "State-of-the-art review on power grid resilience to extreme weather events: Definitions, frameworks, quantitative assessment methodologies, and enhancement strategies," Applied Energy, vol. 239, p. 1049-1065, 2019.

[28] NGESO, “Operating a Low Inertia System,” 2020.

[29] M. Nedd, K. Bell and C. Booth, "Containing loss risk in a low inertia GB power system," in IEEE International Conference on Environmental and Electrical Engineering, Piscataway, 2018

[30] ENA, "Accelerated Loss of Mains Change Programme," [Online]. Available: https://www.energynetworks.org/electricity/engineering/acc elerated-loss-of-mains-change-programme.html. [Accessed 2 May 2020].

[31] NGESO, "System Operability Framework: Low Frequency Demand Disconnection," 2017.

[32] Ofgem, "9 August 2019 power outage report," 2020. 